\title{
Demodulator That Converts Encoder Stereo Signal Into L And R Audio Signal
}

\author{
M. Ibrahim Ashari a,1,*, Ahmad Faisol a,2 \\ ${ }^{a}$ Electrical Engineering, National Institute of Technology Malang, Indonesia \\ ${ }^{1}$ Ibrahim_ashari@lecturer.itn.ac*; ${ }^{2}$ mzfaiz@gmail.com \\ * corresponding author
}

\section{ABSTRACT (10PT)}

Keywords FM_Radio Stereo Decoder
As technology develops in today's age, there are many radio equipment easily found in the market. Certainly, there is a stereo decoder module inside a FM radio receiver which operates to return radio signal that previously was modulated and broken apart into stereo audio signal $(\mathrm{L}+\mathrm{R})$ and $(\mathrm{L}-\mathrm{R})$. There are many stereo decoders already presented inside FM radio receivers. Based on this premise, there is a problem that can be studied, which is how to design a decoder stereo in a stereo FM radio receiver that functions as the demodulation in stereo FM radio and operates to release $\mathrm{L}+\mathrm{R}$ audio signal to a receiver radio. Therefore, it needs a signal demodulator of encoder stereo that converts $\mathrm{L}$ and $\mathrm{R}$ audio signal. Based on the testing result, it showed that sideband frequency from the result of DSB-SC demodulator process, which was produced through $\mathrm{R}$ and L channels, still had frequency value differences with average percentage of $1.416 \%$ out of the expected frequency value. This was due to the frequency oscillator from the generator function.

\section{Introduction}

Telecommunication world has developed rapidly either in brand new invention or even modification. From a point of view of hardware or supporting software, society's need for convenience in communication also involves in telecommunication advancement.

However, all that is strongly associated with the basic component of that said telecommunication. Every basic component is created with the best specification in order to produce high quality and modification in every component is performed in a purpose to make the distinction/identity of a product.

As in component system development in FM radio receivers that keep on developing, there are various component blocks, such as antenna, decoder, audio system, and others which support a receiver radio system[1][2], within the radio system.

As technology develops in today's age, there are many radio equipment easily found in the market. Certainly, there is a stereo decoder module inside a FM radio receiver which operates to return radio signal that previously was modulated and broken apart into stereo audio signal (L+R) and (L-R).

Based on that said premise, the researcher proposed an idea of "DEMODULATOR THAT CONVERTS ENCODER STEREO SIGNAL INTO L AND R AUDIO SIGNAL" in order to complete a demodulation device in a stereo FM radio receiver.

Based on the above description, there are several problems that can be studied further, which are How to design and create a demodulator for converting encoder stereo signal into $\mathrm{L}$ and $\mathrm{R}$ in a receiver radio device. The purpose of this research is to design a demodulator that converts encoder stereo signal into $\mathrm{L}$ and $\mathrm{R}$ audio signal. 


\section{The Proposed Method/Algorithm}

\subsection{Wideband dan Narrowband FM}

When signal is the frequency of modulation, the carrier frequency will shift in line with modulation. This is called deviation. As the same way that modulation level can be varied for modulated signal amplitude, the same way applies to modulated frequency one, even though it is not maximum or modulation level of $100 \%$ as in AM.

Modulation level is set by a number of factors. The available bandwith is one of them. This also shows that signal with big deviation may supports high quality transmission, although they sit on much bigger bandwith[3][4][5].

Signal that has 8 level of low deviation is called Narrow Band Frequency Modulation (NBFM) and it has deviation level of $\pm 3 \mathrm{kHz}$ or more which is used depending on the available bandwith. While wideband has bigger deviation which is $\pm 75 \mathrm{kHz}$.

\subsection{Maintaining the Integrity of the Specifications}

FM receiver must be susceptible to frequency variation of incoming signal in order to be able to receive signal well. As it is known that the width of information band is fluctuative. Therefore, it is made to be susceptible to variation of amplitude, and this may be achieved by adding high gain from IF amplifier. Here, signal is amplified in such a way, and with this method, every variation of amplitude may be erased[6].

Demodulator must depend on frequency in order to be able to convert variation of frequency into variation of voltage. The ideal response is the perfect linear voltage for frequency characteristic. The ideal response cannot be achieved since every system has limited bandwith and as the result, the response curve, which is known as "S" curve, is obtained. This can be viewed that variation of signal frequency is converted into variation of voltage which is able to be amplified by audio amplifier before it enters the device.

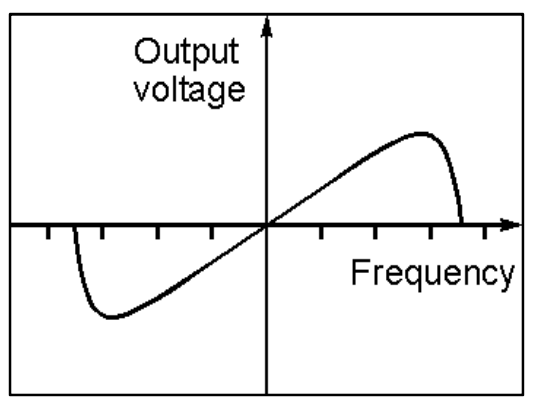

Fig. 1. "S" Curve Characteristic in FM Demodulator

\subsection{FM Demodulator}

There are several circuits which may be used to demodulate FM. Every type has its own advantages and disadvantages, some are used when receiver uses discrete components, and the rest uses IC. Considering how FM usage escalates, even with competition from digital mode that is widely used today, FM demodulation is needed in various new designs[6]

\subsection{Analog Phase Detector}

Phase detector is needed to count the difference of phase from two input signal. It can be likened that $\alpha$ is to be the first input phase and $\alpha$ is the second input phase. The actual input signal to phase detector is in a form of sinus like $\sin (\alpha)$ and $\cos (\beta)$. The first term gives the phase distinction wanted. The second term is sinusoid at twice the reference frequency, so that it is able to be filtered[7]. In the case of common wave of output phase is illustrated with phase detector characteristic. 


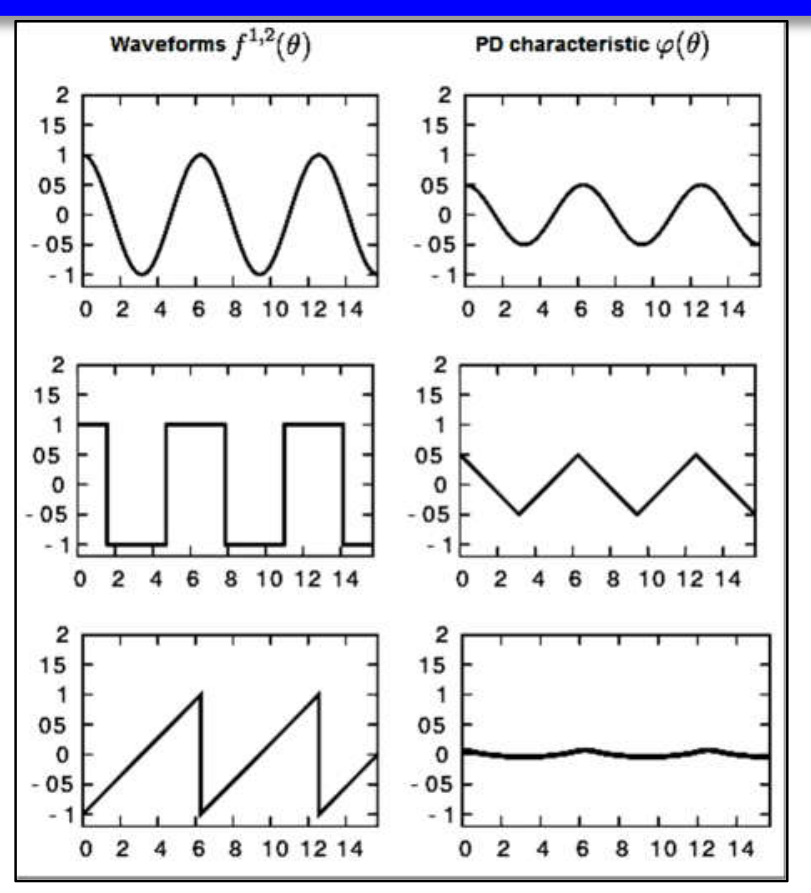

Fig. 2. Phase Detector Multiplier Characteristic

\subsection{Phase Frequency Detector}

A sequence of phase frequency detector is a sequence of asynchronous sequential logic which is initially made of four flip-flop (it is a phase frequency detector found in both IC RCA CD4046 and MC4344 in 1970)[8]. The logic decides which one out of two signals that have zero-crossing more premature or more often. When it is applied in PLL application, lock may be achieved. The detector has an advantage which produces output even when two signal compared are not only in a phase, but also in frequency. A phase frequency detector prevents a condition (false key) in PLL application where PLL synchronizes with the false phase out of input signal or with the false frequency.

A phase value that is irregular is going to be weaken by a pulse detector to produce value that is regular, either positive or negative, for a capacitor to act as an integrator.

A proportional phase detector works out proportional value with detected false phase. Some have deadband and some do not. Specifically, some designs produce back up and pulse down control even when phase distinction is zero. This pulse is small, has the same duration and causes value weaken in order to produce the same pulse, either positive or negative current fits for this phase. A phase detector with control system type does not show a deadband and usually has lower minimum jitter (peak to peak) if it is applied in PLL.

In PLL application (phase locked loop) often is required to figure out when loop is locked. A digital phase frequency detector that is more complex typically has output that allows reliable indication out of locked condition.

\subsection{Implementation and Appalication of Schmitt Trigger}

A circuit with hysteresis is based on basic aide of positive feedback. Every active circuit is able to be created to act as the trigger by applying positive feedback, so that loop gain more than one. Positive feedback is introduced by adding a part from output voltage for input voltage. Therefore, this circuit contains attenuator. 


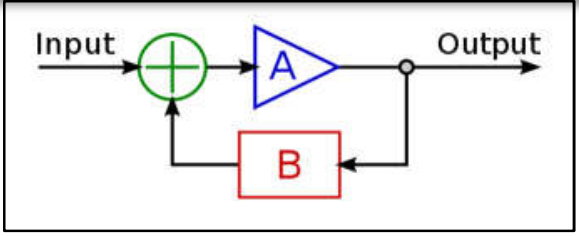

Fig. 3. Ideal Feedback of Schmitt trigger

\section{Method}

\subsection{FM Stereo Block Diagram of Multiplex Demodulation}

Block diagram overall described how the sequence work later as it should and this block diagram later will interconnect with stereo decoder sequence which are built by using IC AN7410N[9].

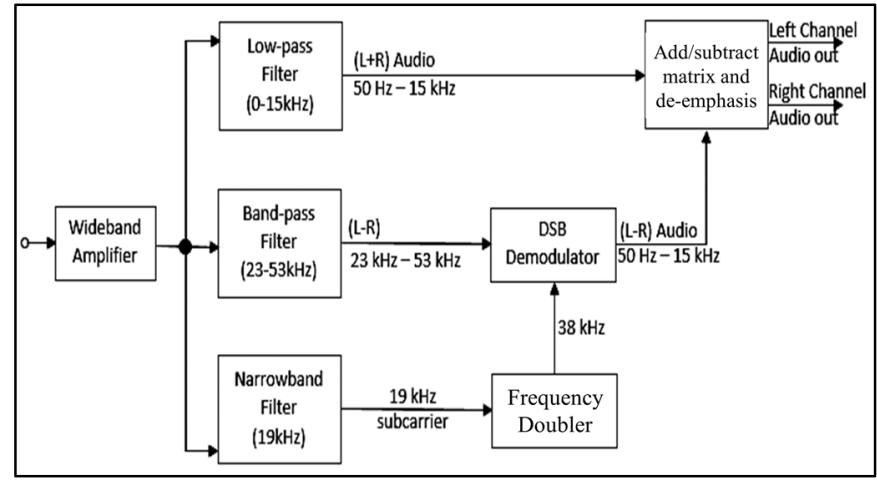

Fig. 4. System Block Diagram

The explanations of block diagram are below:

- Wideband Amplifier

Wideband Amplifier is a circuit that provides amplification constantly with frequency ratio of low angle to high angle frequency for more than one octave. Wideband amplifier tends to be complementary in a concept for audio amplifier.

- Low-pass Filter $(0-15 \mathrm{kHz})$

Low-pass Filter erases all frequency which excesses $15 \mathrm{kHz}$ and certainly has the amount of signal $(\mathrm{L}+\mathrm{R})$ in output. In this receiver, it is going to be the only output getting processed further through de-emphasis for audio amplification.

$$
f C=\frac{1}{2 \pi R C}
$$

LPF (low pass filter) in this stereo decoder has frequency range from $50 \mathrm{~Hz}$ to $15 \mathrm{kHz}$ as a reference from audio signal $(\mathrm{L}+\mathrm{R})$. Thus, a cut-off frequency value needs to be determined beforehand with below calculation:

For resistor value of $1 \mathrm{~K} \mathrm{Ohm}$ and capasitor $220.000 \mathrm{pF}$

$$
\begin{aligned}
& \omega c=\frac{1}{R C} \\
& \omega c=\frac{1}{\left(1 \times 10^{3}\right)\left(220.000 \times 10^{-12}\right)} \\
& \omega c=\frac{1}{0.00022} \\
& \omega c=4545,4545 \mathrm{~Hz}
\end{aligned}
$$




$$
\begin{aligned}
& f c=\frac{\omega c}{2 \pi} \\
& f c=\frac{4545,4545}{2 \times 3.14} \\
& f c=723,79 \mathrm{~Hz}
\end{aligned}
$$

- Band-pass Filter (23-53 kHz)

Band-pass filter selects sideband that is suitable with discrete signal or differences between two channels (L-R) and also reject optional frequency of SCA above $59.5 \mathrm{kHz}$. Sideband is fed to a detector source or to balance modulator which also receives output from frequency doubler. Frequency carrier of $38 \mathrm{kHz}$ is suppressed in order for sideband which carries of $38-53 \mathrm{kHz}$ and $23-38 \mathrm{kHz}$ to have more roles in a transmitter deviation. Since this $38 \mathrm{kHz}$ carrier is required to detect subcarrier, $38 \mathrm{kHz}$ signal must be available in FM stereo. In order to confirm synchronization, this $38 \mathrm{kHz}$ carrier is created with $19 \mathrm{kHz}$ pilot carrier because $19 \mathrm{kHz}$ signal is out of the range of audio frequency $(\mathrm{L}+\mathrm{R})(0-15 \mathrm{kHz})$ as well as the range of subcarrier frequency of 23-53 kHz. This simplifies recovering the subcarrier without interference from audio signal.

- Narrowband Filter (19 kHz)

Doubler converts carrier signal of $19 \mathrm{kHz}$ (pilot tone), which is selected with narrowband filter for carrier signal of $38 \mathrm{kHz}$, which is later going to be amplified. Amplitude of carrier signal (subcarrier) has been reduced. Signal or frequency that is other than $19 \mathrm{kHz}$ is going to be ignored because narrowband will only pass $19 \mathrm{kHz}$ as the stereo signal detection.

- Frekuensi Doubler dan Amplifier

Carrier signal (subcarrier) of $19 \mathrm{kHz}$ that has been previously processed in narrowband filter is going to be processed in frequency doubler which means carrier signal will be multiplied and amplified in order to produce $38 \mathrm{kHz}$ signal. Next this is going to be processed in DSB Demodulator.

- DSB Demodulator

Both input from frequency double and band-pass filter within the SSB demodulator in this circuit produce desired discrete signals (L-R). If they are fed on matrix along with (L+R), it produces left channel from doubler and the right channel from a subtractor. After de-emphasis (muffling noise), audio will be amplified further.

- Add/subtract Matrix and De-emphasis

Both input from the result of DSB demodulator in this circuit produce desired discrete signals (L-R). If they are fed on matrix along with $(\mathrm{L}+\mathrm{R})$, it produces left channel from doubler and the right channel from a subtractor. Finally, SCA signal is obtained, demodulated, de-emphasis, and produced as separated audio output which is left channel and right audio.

\subsection{IC AN7410N Block Diagram}

From previous block diagram FM stereo multiplex demodulation, it is going to be applied into a sequence which are within IC AN7410N and obtained several test points between IC AN7410N and FM stereo multiplex demodulation. 


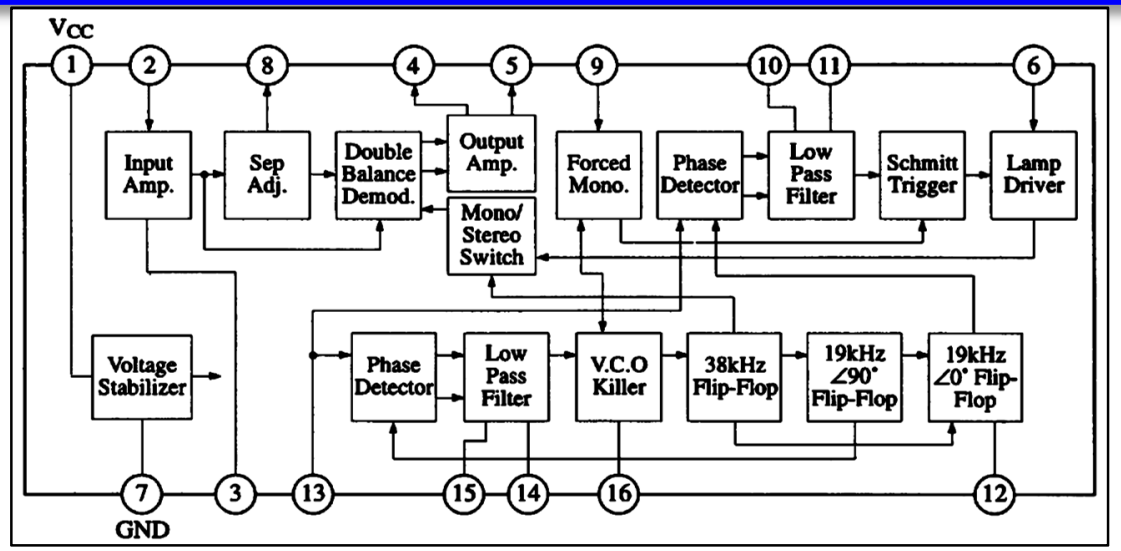

Fig. 5. AN7410N Block Diagram

The explanations for block diagram above are below:

- Voltage Stabilizer It is an input voltage stabilizer (VCC) with maximum 12V DC by using power supply which is connected to the ground (GND).

- Input Amp. It $s$ an input from stereo signal (stereo signal input) wherein that stereo signal is going to be amplified first. In other words, Input Amp. is a wideband amplifier as the amplification that works constantly with frequency ratio of low angle to angle frequency higher than one octave.

- Separation Adj. It operates to separate input signal from input amp. (wideband amplifier) and the signal will be fed further on double balance demodulator.

- Double Balance Demodulator. It functions as a rectifier or balancer from input signal which later it is going to be out of output amp. and re-amplified and detected whether that signal is mono or stereo. If the signal is detected as mono, then it is going to be ignored.

- Output Amp. It is the signal result from Doubel Balance Demodulator which has already been rectified and amplified as well as forwarded to determine whether that signal is mono or stereo which it will be visible with LED indicator.

- Mono/Stereo Switch. It is a detector to figure out whether the input signal is mono or stereo. If it is detected that there is $19 \mathrm{kHz}$ pilot tone, which indicates that there is stereo signal, then an indicator lamp is going to be on, but if the signal is detected as mono, then it is going to be ignored.

- Forced Mono. It is a place for mono signal (not stereo) which later is going to be dumped by VCO killer or that signal is considered non-existent.

- VCO Killer (voltage control oscillator) operates to determine work frequency of oscillator. Its input comes from voltage (input amp.) and its output is in the form of the result frequency of PLL processing which is the expected frequency.

- Phase Detector. It is a frequency mixer, analog multiplier or circuit logic that produces voltage signal which represents phase distinction between two input signals and acts to detect phase distinction from two input signals and produces constant output signal.

- Low Pass Filter. It operates to pass by output with frequency below the cut-off frequency and to weaken or dump output voltage to unwanted certain frequency. It can also mean that to erase all frequency above $15 \mathrm{kHz}$ and certainly the one that has the amount signal $(\mathrm{L}+\mathrm{R})$ in output with frequency range between $50 \mathrm{~Hz}$ and $15 \mathrm{kHz}$. In this receiver, it is going to be the only output that is going to be processed further through de-emphasis for audio amplification.

- Schmitt Trigger. It is a sequence of comparator by applying positive feedback to non-inverting input from comparator or differential booster. This sequence names a trigger because the output 
maintains its value until the modification input is enough to trigger modification. In noninverting configuration, when the input is higher than the chosen threshold, then the output will be high. When the input is lower than the threshold, then the output is low, and when the input is in two levels above the output, then the output keeps maintaining its value. This is also called as hysteresis and it indicates that Schmitt trigger has memory and is able to act as bistable circuit (latch or flip-flop)[10].

$$
V+=\frac{\mathrm{R} 2}{\mathrm{R} 1+\mathrm{R} 2} \cdot \mathrm{Vin} \frac{\mathrm{R} 1}{\mathrm{R} 1+\mathrm{R} 2} \cdot \mathrm{Vs}
$$

Schmitt trigger is applied in signal conditioning application to eliminate noise from signal used in the sequence.

- Lamp Driver. It is a LED (Light Emitting Diode) or an indicator to figure out the signal whether it is mono or stereo. LED lamp will only light up if it receives $19 \mathrm{kHz}$ frequency (pilot tone) as a sign that the signal received is stereo signal.

- $38 \mathrm{kHz}$ Flip-flop. It is a carrier signal that blends with sound signal (voice) which operates to detect $38 \mathrm{kHz}$ subcarrier signal and produce $38 \mathrm{kHz}$ precision signal which is used for demodulation process or to separate additional signal in order to be audio signal (R-L).

- $19 \mathrm{kHz}$ Flip-flop $90^{\circ}$ It is the division result of $38 \mathrm{kHz}$ subcarrier signal to be $19 \mathrm{kHz}$ subcarrier signal. $19 \mathrm{kHz}$ frequency here experiences phase shift of $90^{\circ}$.

- $15.19 \mathrm{kHz}$ Flip-flop $0^{\circ}$. It is the division result of $38 \mathrm{kHz}$ subcarrier signal to be $19 \mathrm{kHz}$ subcarrier signal. $19 \mathrm{kHz}$ frequency here does not experience phase shift or in other words, the phase shifts $0 \mathrm{o}$.

\section{Results and Discussion}

\subsection{DSB-SC Demodulator Testing}

This DSB-SC testing applied two different frequency input with the assistant of Function Generator, which would generate frequency from $36 \mathrm{kHz}$ to $40 \mathrm{kHz}$ as the subcarrier frequency, and Auda City software to generate $19 \mathrm{kHz}$ pilot frequency. Output frequency would be monitored by using Frequency counter for each output in the audio channel either $\mathrm{R}$ audio channel or L audio channel.

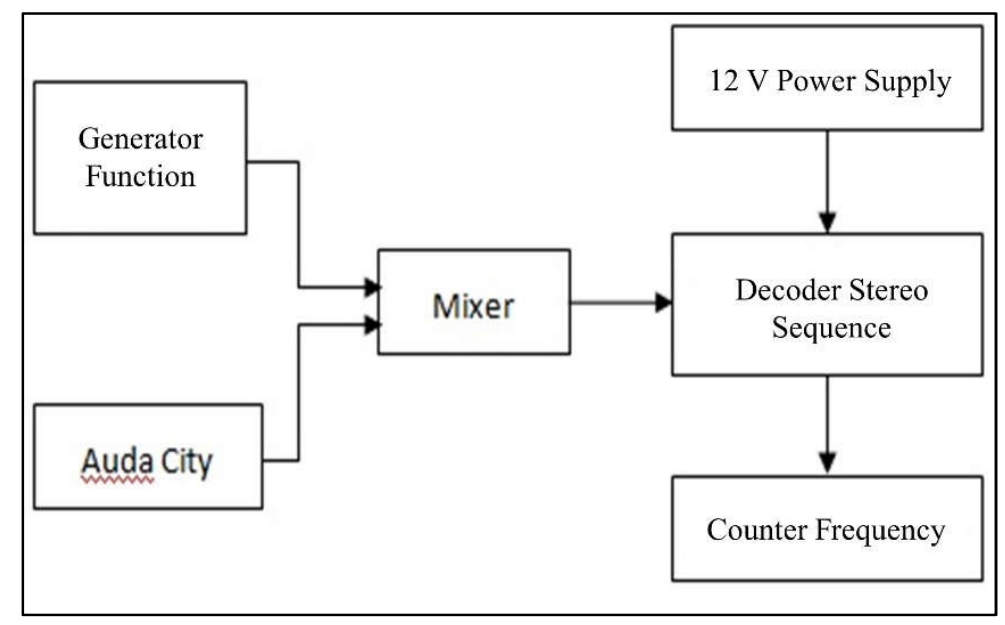

Fig. 6.. Block Diagram of DSB-SC Demodulator Testing

Fig. 7 depicts the frequency result out of $\mathrm{R}$ audio channel output from decoder sequence if the Function Generator was set in $36 \mathrm{kHz}$. 


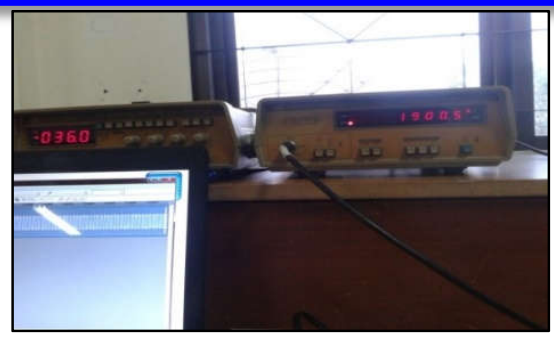

Fig. 7. Frequency Result of R Audio Channel in $36 \mathrm{kHz}$ Frequency

Figure 8 depicts the frequency result out of L audio channel output from decoder sequence if the Function Generator was set in $36 \mathrm{kHz}$.

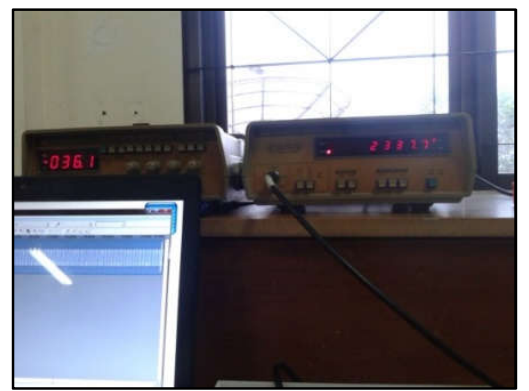

Fig. 8. Frequency Result of L Audio Channel in $36 \mathrm{kHz}$ Frequency

The following is a table for testing result of output frequency in each audio channel against subcarrier frequency.

Table 1. Frequency Output Result in L Audio Channel

\begin{tabular}{cc}
\hline Input Frequency (kHz) & Output Frequency (Hz) \\
\hline $36 \mathrm{kHz}$ & 2337.7 \\
\hline $37 \mathrm{kHz}$ & 1019.6 \\
\hline $38 \mathrm{kHz}$ & 50.1 \\
\hline $39 \mathrm{kHz}$ & 1107.8 \\
\hline $40 \mathrm{kHz}$ & 1772 \\
\hline
\end{tabular}

Table 2.

Tabel 2. Hasil keluaran frekuensi pada kanal audio R

\begin{tabular}{cc}
\hline Input Frequency (kHz) & Output Frequency (Hz) \\
\hline $36 \mathrm{kHz}$ & 1900.5 \\
\hline $37 \mathrm{kHz}$ & 1038.6 \\
\hline $38 \mathrm{kHz}$ & 50 \\
\hline $39 \mathrm{kHz}$ & 907 \\
\hline $40 \mathrm{kHz}$ & 1593.6 \\
\hline
\end{tabular}




\subsection{Testing Result Analysis}

Based on the testing result of output frequency from either L or R audio channel, output frequency has small differences from the desired frequency.

\subsection{Encoder to Decoder Testing}

This encoder to decoder sequence testing is to figure out synchronization from both sequences wherein encoder acted as the audio signal sender and decoder as the audio signal receiver. The testing also aimed to figure out whether the signal produced from modulation and demodulation process was stereo with frequency input which its frequency result would be scanned by the frequency counter.

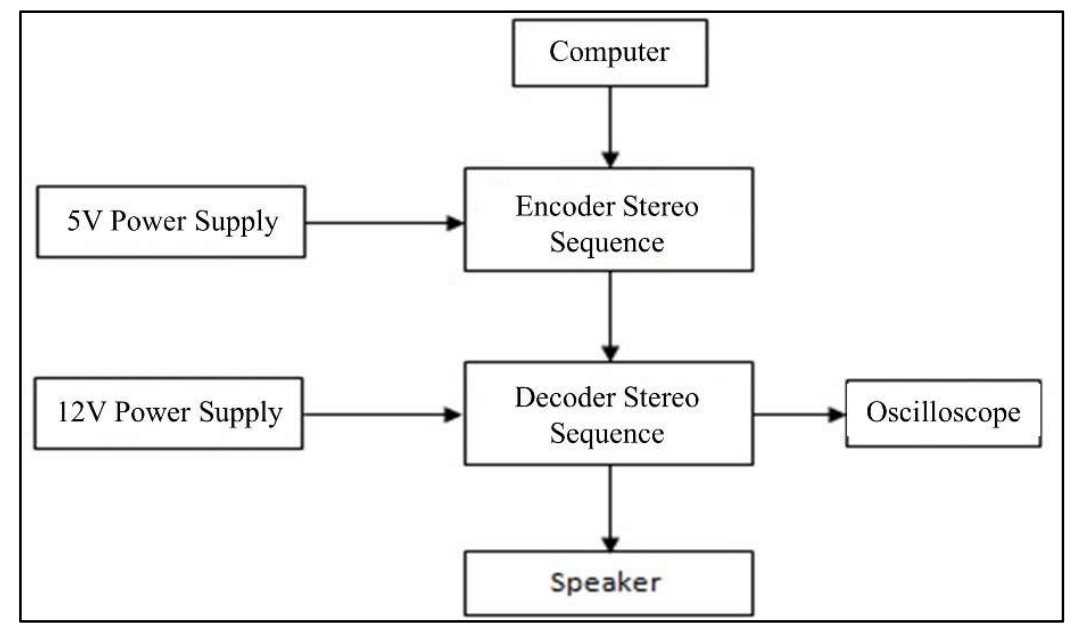

Fig. 9.. Block Diagram of Encoder to Decoder Testing

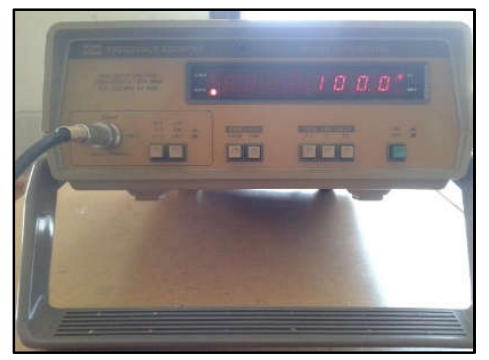

Fig. 10. Output Frequency Result in L Audio Channel

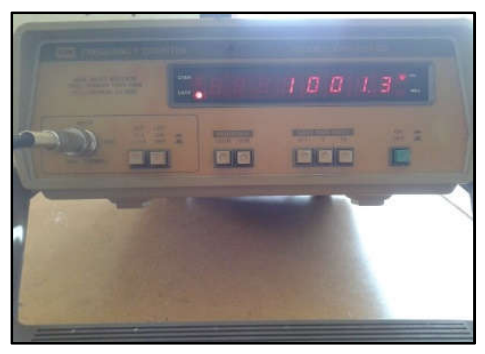

Fig. 11. Output Frequency Result in R Audio Channel

\subsection{Testing Result Analysis}

Based on the testing result that has been done, it was generated that encoder and decoder stereo sequences were well-connected from which the audio produced already showed audio result of stereo. Decoder stereo sequence was able to detect pilot tone produced by encoder stereo sequence with lighted up LED lamp and $19 \mathrm{kHz}$ frequency result was depicted in oscilloscope digital.

Also, the stereo testing used frequency as the input signal that was inputted into each channel in encoder stereo sequence then its output frequency was read in decoder stereo which L channel had 
output frequency that was suitable with its input frequency $(100 \mathrm{~Hz})$, while the output frequency result in $\mathrm{R}$ channel had frequency differences of $1.3 \mathrm{~Hz}$ out of input frequency $(1 \mathrm{kHz})$.

\section{Conclusion}

After the design process, setting up, and device testing were performed, several conclusions were taken as Decoder stereo sequence was already detected $19 \mathrm{kHz}$ pilot tone frequency which was revived either through software assistance or FM tunner. Radio broadcast signal was able to be detected by decoder stereo sequence. Sideband frequency which was the result of DSB-SC demodulator which was produced through $\mathrm{R}$ and $\mathrm{L}$ channel was still having differences in frequecny value with average percentage of $1.416 \%$ out of expected frequency value. This was due to the frequency oscillator from generator function. Decoder stereo sequence produced stereo audio. Even though there were some lacks in the demodulation process and audio produced, decoder stereo sequence still function well.

\section{References}

[1] U. Kurniawan, "Pengantar Telekomunikasi," Informatika, 2010.

[2] A. E. Putra, "Belajar Mikrokontroler AT89C51/52/55 Teori dan Aplikasi," Gava Media, Yogyakarta, 2002.

[3] D. Roddy, J. Coolen, and K. Idris, Komunikasi Elektronika. Erlangga, Jakarta, 1986.

[4] R. Arifuddin, D. R. Santoso, and O. Setyawati, "Pengembangan Sistem Akuisisi Data Nirkabel untuk Pengukuran Distribusi Suhu Bawah Permukaan,” J. EECCIS, vol. 9, no. 2, pp. 123-129, 2016.

[5] P. D. P. Adi and R. Arifuddin, "Design Of Tsunami Detector Based Sort Message Service Using Arduino and SIM900A to GSM/GPRS Module," JEEMECS (Journal Electr. Eng. Mechatron. Comput. Sci., vol. 1, no. 1, pp. 1-4, 2018.

[6] F. B. Paimin, Membuat dan mengelola mesin tetas. Penebar Swadaya Grup, 1992.

[7] A. P. Malvino, "Prinsip-Prinsip Elektronika Jilid 1," Penerbit Erlangga: Jakarta, 1996.

[8] G. Jong Bloe, "Elektronika Merencanakan dan Merakit Sendiri.” Angkasa Bandung, Bandung, 1998.

[9] Internet:ww.cytron.com.my. Download HSM-20G data sheet.

[10] Internet: www.semiconductor.philips.com download PCF9591datasheet 